\title{
Towards Universal GAN Image Detection
}

\author{
Davide Cozzolino, Diego Gragnaniello, Giovanni Poggi, Luisa Verdoliva \\ University Federico II of Naples, Italy \\ Email: name.surname@unina.it
}
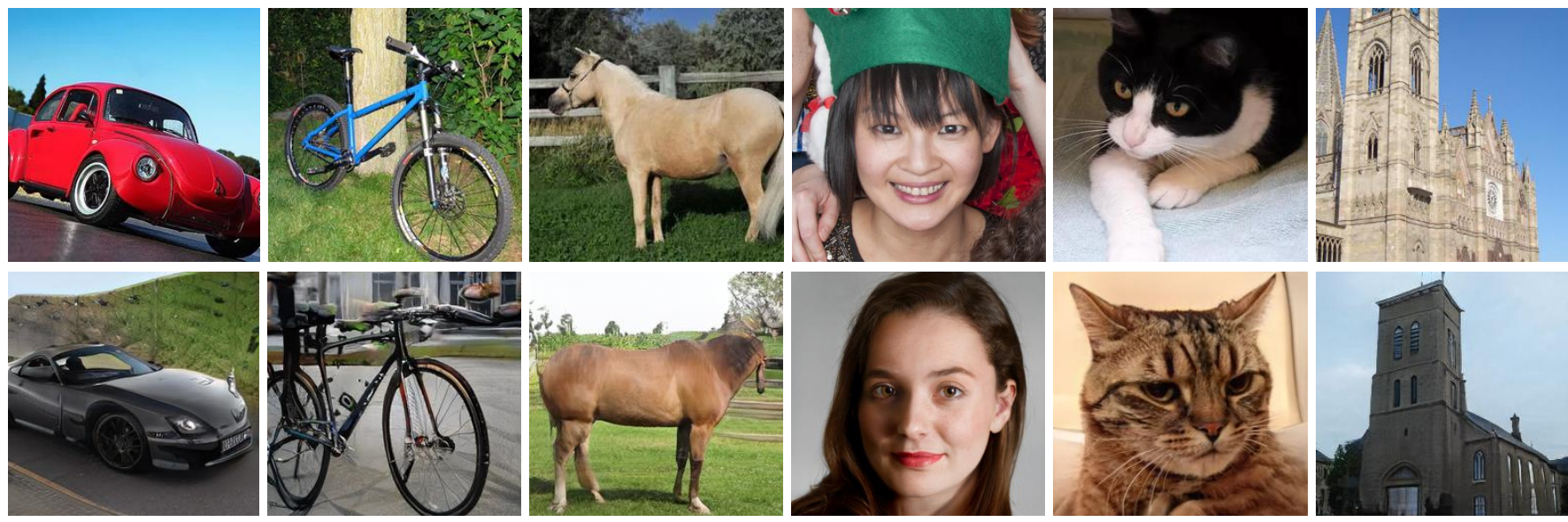

Fig. 1: Pristine (Top) and synthetic (Bottom) images generated using ProGAN [1] and StyleGAN2 [2] architectures.

Abstract-The ever higher quality and wide diffusion of fake images have spawn a quest for reliable forensic tools. Many GAN image detectors have been proposed, recently. In real world scenarios, however, most of them show limited robustness and generalization ability. Moreover, they often rely on side information not available at test time, that is, they are not universal. We investigate these problems and propose a new GAN image detector based on a limited sub-sampling architecture and a suitable contrastive learning paradigm. Experiments carried out in challenging conditions prove the proposed method to be a first step towards universal GAN image detection, ensuring also good robustness to common image impairments, and good generalization to unseen architectures.

\section{INTRODUCTION}

Image synthesis based on GAN technology has apparently reached full maturity. Two recent studies [3], [4] have independently proved that humans cannot reliably tell apart images generated by advanced GAN technologies, such as StyleGAN2 [2], from pristine images. The average accuracy turned out to be around $50 \%$ (coin tossing) for untrained observers, increasing to just $60 \%$ for trained observers with unlimited analysis time [3]. This raises legitimate concerns about the use of GAN images for the most different purposes. Besides plain jokes, such as the fictional profile of a U.S. Congress candidate built on Twitter [5] by a seventeen years old kid, there is also the growing spread of government propaganda over Facebook [6]. In addition, while early efforts were almost exclusively on faces, recent GANs can realistically generate from scratch many other categories, as shown in Fig.1 where real and high-quality synthetic images are shown.

Yet, while GAN images can evade the scrutiny of human observers, their generation process leaves distinctive traces [7],
[8] which allow detection by means of dedicated forensic tools. In the literature, there has been an intense research effort for reliable GAN image detectors [9], and many of them have shown impressively good results when suitably trained.

However, one of the main challenges in GAN image detection is generalization. In fact, if a detector is tested on the very same type of images seen in the training phase it will hardly fail. Unfortunately, this is not what happens in a realistic scenario. To operate successfully in the wild, a detector should $i$ ) be robust to image impairments, ii) work universally well across sources and iii) generalize well to new sources. Robustness to image impairments is essential, since most social networks resize and compress images to satisfy their internal constraints. These non-malicious operations destroy precious evidence, with a possible detrimental effect on detection performance. Universality is also a fundamental requirement, since the detector ignores the image source (which GAN architecture was used to generate it) and cannot use this information. Finally, the image under test may have been generated by a totally new architecture, and the detector should work well also in this condition.

The issue is touched upon in [10] where it has been also shown that a neural network trained to detect images generated by a given GAN generalizes well to image generated by other GANs, provided suitable augmentation is used in training. Experiments prove the proposed detector to ensure a large AUC (area under the ROC curve) figure on GAN images never seen in training. The AUC, however, measures the potential of the method rather than its actual performance. A large AUC ensures that scores for real and fake images are reasonably separable, and reliable detection is possible given a good 
decision threshold. However, the decision threshold itself is unknown and, more important, it varies largely from GAN to GAN. In [10] it is observed that a good decision threshold can be estimated even from just two images, one real and one fake. However, in real-world testing conditions, the detector does not know which GAN possibly generated the image under test and hence which threshold to use.

Here, we propose a contrastive learning based architecture that ensures a more stable performance across different sources. Experiments carried out in real-world conditions, with performance metrics less forgiving than the AUC, show the proposed detector to outperform all reference methods.

\section{RELATED WORK}

In recent years, there has been intense research on forensic detectors that distinguish between real and synthetic content. Leveraging the opportunity to generate large datasets of GAN images, the most effective techniques work in a supervised setting and achieve excellent results both working in the spatial [11], [12] and in the frequency domain [13], [14]. These detectors provide an excellent performance in ideal conditions, that is, with high-quality images that come from a GAN architecture included in the training set. Often, however, the performance degrades sharply in more challenging realworld scenarios, with images that are resized and compressed, and may be generated by GAN architectures that were not included in training. To improve generalization, [15] proposes a few-shot learning method, while an approach based on incremental learning is considered in [16]. However, in both cases the improved generalization is obtained by exploiting a few example images from the new GAN architectures, pieces of information hardly available in a real-world scenario.

The most successful tool to gain both generalization and improved robustness is augmentation. In [17] the idea is to carry out augmentation by Gaussian blurring, so as to force the detector to learn more general features while discarding noise-like patterns that impair the training. A similar approach is followed in [10] where a standard pre-trained model, ResNet50, is further trained with a strong augmentation based on compression and blurring. Experiments show that, even when the detector is trained on images from a single GAN architecture, the learned features generalize well to unseen architectures, datasets, and training methods. This approach is further analyzed in [18] where several variations are considered. In particular, inspired by recent work in steganalysis [19] and image forensics [20], an architecture where downsampling operations are removed from the first layer of the network is proposed. The promising results confirm the need to preserve the subtle traces hidden in high-frequency image components for forensic applications.

\section{PROPOSED METHOD}

Here we propose a new GAN image detector aimed at ensuring good generalization and robustness also in real-world scenarios. We build upon the lessons learnt in [10] and in [18]

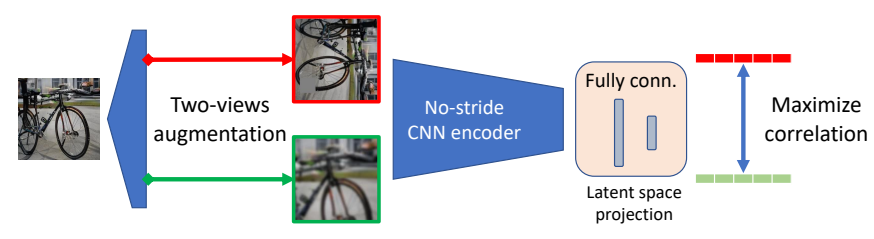

Fig. 2: Scheme of our contrastive learning based approach.

and accordingly try to $i$ ) avoid early subsampling of input data, and $i$ ) use intense augmentation of the training data.

In terms of architecture, we use the same backbone adopted in [10], a simple ResNet50 pretrained on ImageNet but, as proposed in [18], we do not perform subsampling in the first layer. As for augmentation, we turn to a contrastive learning paradigm [21], [22], suitably adapted to our peculiar needs. More specifically, we adopt the two-step training procedure proposed in [21] for visual representation learning. We begin by removing the last fully-connected layers from our ImageNet pre-trained backbone. The feature vector computed by the network after the global average pooling is then projected in a new latent space for a first self-supervised training phase. This crucial step aims at extracting a representation of the input image that is as invariant as possible to various forms of distortion and impairment. To this end, the network is fed with multiple augmented versions of the same image, and forced to generate similar latent vectors for them by minimizing a suitable contrastive loss. In particular, we use the Normalized Temperature-scaled Cross Entropy (NT-Xent) loss proposed originally in [21]. Let $u$ and $u^{+}$be the latent vectors extracted from two different augmented versions of the original image $\mathrm{I}$, and let $v$ indicate either the latent vector $u^{+}$or a generic latent vector extracted from another image, unrelated to I, then

$$
\text { Loss }=-\operatorname{sim}\left(u, u^{+}\right) / \tau+\log \sum_{v \neq u} \exp (\operatorname{sim}(u, v) / \tau)
$$

Here, $\operatorname{sim}(u, v)=u^{T} v /(\|u\|\|v\|)$ is the cosine similarity (the dot product between normalized $u$ and $v$ ), the summation on $v$ includes the compact feature vectors extracted by all images of the batch except $u$, and $\tau$ is a temperature parameter, set to 0.07 in our experiments. By minimizing the NT-Xent loss, the network increases the correlation between $u$ and $u^{+}$as opposed to the correlation between $u$ and the other latent vectors of the batch.

This approach fits very well our needs, and we use a large set of augmentations which reproduce most of the postgeneration processing applied to synthetic images, for example when they are posted on a social network. Beyond JPEG compression and Gaussian blurring, already adopted in [10], we also randomly vary the brightness, contrast, saturation, and hue, as well as random gray-scale conversion. Also, images are randomly distorted adding Gaussian noise or removing patches via CutOut. Then, random patches of dimension $96 \times 96$ are extracted during training. Instead, at test time we perform inference on full-resolution images thanks to the global average pooling. It is worth emphasizing that both at 
training and at test time no resizing is carried out in order to preserve information and avoid destroying important traces.

Subsequently, we add the fully-connected layers and finetune the network in a supervised manner for our final task, that is synthetic image detection. Note that, unlike in [21], we fine-tune the whole network and not only the last layers. In fact, we are interested in all the low- and mid-level traces left by the GANs in the generation process, and not only to high-level visual representation.

As for the implementation details, to project the image representation in the latent space for the self-supervised learning, we use two fully-connected layers of size 2048 and 128, interleaved by rectified linear unit activation. During the selfsupervised training, the network parameters are updated via stochastic gradient descent with an initial learning rate of 1e4 , then reduced by a factor of 10 until $1 \mathrm{e}-6$ if the validation loss does not decrease after 5 consecutive epochs, and a batch size of 64 images ( 2 different views obtained from 32 original images). Finally, during the supervised fine-tuning, the Adam [23] optimizer is adopted with standard parameters, an initial learning rate of $1 \mathrm{e}-5$ and the same learning rate scheduling and batch size of the self-supervised training.

\section{EXPERIMENTAL RESULTS}

In this Section we report the experimental results of our method and show comparisons with state-of-the-art. For a fair comparison, we trained both our proposal and the competitors on the same single dataset. This allows to easily test the generalization ability of the CNN models. More precisely, we use the training set provided in [10] comprising $362 \mathrm{~K}$ real images extracted from various object categories of the LSUN dataset and $362 \mathrm{~K}$ images generated by 20 ProGAN [1] models. All images have the same resolution of $256 \times 256$ pixels.

To test generalization, we adopt the same setting considered in [18] and include both low resolution $(256 \times 256)$ and high resolution $(1024 \times 1024)$ images coming from GAN architectures never seen in training: StyleGAN [24], StyleGAN2 [2], BigGAN [25], CycleGAN [26], StarGAN [27], RelGAN [28], and GauGAN [29]. Overall, we have a total of 39K synthetic images. For the pristine data we consider different sets based on the image resolution: ImageNet, COCO [30], and Unpaired real dataset [26] for low-resolution images and the RAISE dataset [31] for high resolution ones. To avoid any form of polarization, we avoid using the same source of real images (e.g. LSUN) for training and testing. Overall we have a total of $11.1 \mathrm{~K}$ low-resolution and $7.8 \mathrm{~K}$ high-resolution real images.

For comparisons we consider the following state-of-the-art GAN detectors: Spec [13], FFD [11], PatchForensics [12], CNN-DCT [14], Wang2020 [10], no-down [18]. Note that for PatchForensics we use the original weights of the proposal, also obtained by training the network on ProGAN.

Our first set of experiments aims at testing generalization on GAN architectures never seen in training. In Tab [ we report both AUC and Accuracy. Except for CNN-DCT and FFD, all methods ensure very high AUC values, going from 0.888 to 0.997, on the average. However, such large AUC values often

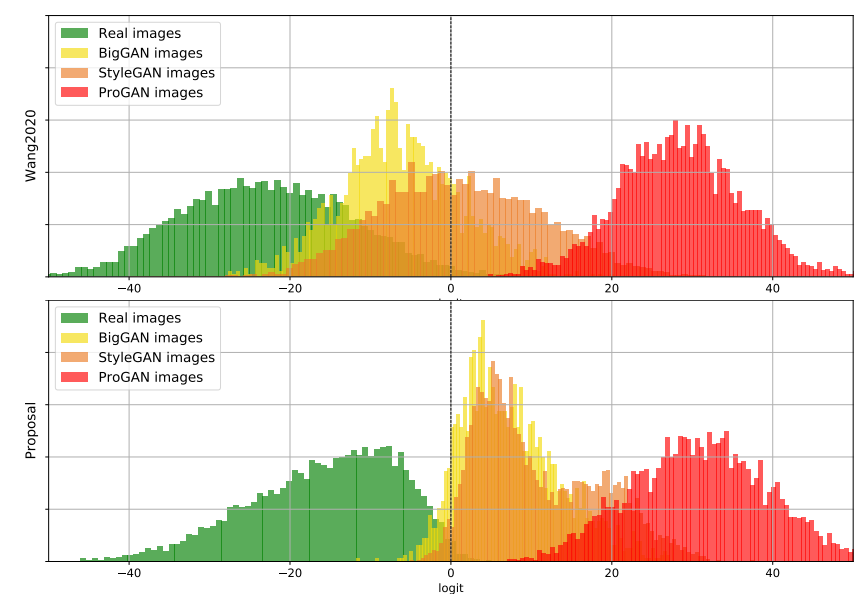

Fig. 3: Distribution of network output. For Wang2020 (top) a different threshold is necessary for each GAN while for the proposed method (bottom) a single threshold works well in all cases.

correspond to very low accuracies (e.g., PatchForensics on StyleGAN). The point is that a large AUC means only that a good threshold exists which separates well synthetic from real images. Note that this threshold is unknown and may vary from case to case. Lacking this prior information, we used a fixed 0.5 threshold in the experiment, obtaining several disappointing results. The problem is well highlighted by the histograms of scores reported in Fig 3. For Wang2020 [10], real and fake images cannot be accurately separated with a single threshold for all cases. On the contrary, for our proposal, a fixed threshold separates well real data from synthetic images coming from different architectures. Accordingly, in Tab 1 the proposed method achieves both high AUC and high accuracy, showing a good generalization ability.

To gain further insights, we also show in TabII the probability of detection obtained by setting the false alarm rate at a fixed value of 10\% (Pd@10\%) and 1\% (Pd@1\%). This is a very appropriate performance measure for real-world operations, where a huge number of images, mostly real, must be analyzed, and a low false alarm rate is mandatory to avoid being swamped by false positives. Note that the threshold implicitly defined by this metric depends solely on the real images. Overall performance drops for most of the compared methods. Acceptable values are obtained only with PatchForensics and Wang2020, with an average Pd@10\% of 80.0 and 88.1 , respectively. On the contrary, a very good performance is obtain with no-down (97.5) and our proposal (99.6) which share the choice of avoiding sub-sampling in the first layers. For the proposed method, even Pd@1\% exceeds 90 , on the average, outperforming all reference methods.

Finally, to test robustness to typical impairments occurring on social networks, we applied post-processing operations like JPEG compression (with different quality factors) and image resizing (at different scales). Results in terms of accuracy are compared in Fig 4. For several methods, performance reduces dramatically in the presence of compression and resizing, and remains almost stable only for methods that use strong 


\begin{tabular}{|c|c|c|c|c|c|c|c|c|}
\hline \multicolumn{2}{|c|}{$\mathrm{ACC} / \mathrm{AUC}$} & Spec & FFD & PatchForensics & CNN-DCT & Wang2020 & no-down & proposed \\
\hline \multirow{8}{*}{$\begin{array}{l}\dot{2} \\
3 \\
0 \\
0\end{array}$} & ProGAN & $78.3 / 0.987$ & $85.1 / 0.977$ & $66.1 / 0.982$ & $60.0 / 0.664$ & $99.3 / 1.000$ & $94.7 / 1.000$ & $99.4 / 1.000$ \\
\hline & StyleGAN & $63.6 / 0.704$ & $70.2 / 0.761$ & $59.2 / 0.959$ & $53.2 / 0.478$ & $75.9 / 0.954$ & $93.7 / 0.988$ & $98.2 / 0.999$ \\
\hline & StyleGAN2 & $53.8 / 0.557$ & $64.6 / 0.726$ & $53.0 / 0.933$ & $29.8 / 0.270$ & $71.5 / 0.946$ & $92.2 / 0.980$ & $89.0 / 0.990$ \\
\hline & BigGAN & $77.4 / 0.929$ & $57.4 / 0.640$ & $52.0 / 0.867$ & $60.0 / 0.697$ & $59.2 / 0.900$ & $93.5 / 0.985$ & $95.3 / 0.996$ \\
\hline & CycleGAN & $78.2 / 0.961$ & $67.8 / 0.733$ & $63.7 / 0.833$ & $60.1 / 0.700$ & $77.4 / 0.968$ & $90.3 / 0.969$ & $94.0 / 0.994$ \\
\hline & StarGAN & $77.0 / 0.819$ & $85.3 / 0.987$ & $98.5 / 1.000$ & $60.1 / 0.703$ & $84.3 / 0.978$ & $94.5 / 0.992$ & $98.6 / 0.999$ \\
\hline & RelGAN & $78.3 / 0.992$ & $85.3 / 0.971$ & $99.3 / 1.000$ & $60.1 / 0.703$ & $63.6 / 0.914$ & $92.8 / 0.977$ & $96.4 / 0.997$ \\
\hline & GauGAN & $76.8 / 0.890$ & $64.5 / 0.727$ & $50.0 / 0.751$ & $60.1 / 0.703$ & $82.5 / 0.975$ & $93.6 / 0.988$ & $94.5 / 0.996$ \\
\hline \multirow{4}{*}{ 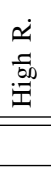 } & ProGAN & $83.3 / 0.936$ & $69.2 / 0.962$ & $97.9 / 1.000$ & $66.9 / 0.668$ & $99.7 / 1.000$ & $97.1 / 1.000$ & $95.3 / 1.000$ \\
\hline & StyleGAN & $87.3 / 0.989$ & $68.0 / 0.877$ & $50.6 / 0.954$ & $59.7 / 0.647$ & $91.0 / 0.993$ & $96.8 / 0.997$ & $95.3 / 1.000$ \\
\hline & StyleGAN2 & $87.8 / 0.999$ & $68.8 / 0.867$ & $50.0 / 0.912$ & $55.5 / 0.540$ & $73.2 / 0.960$ & $96.9 / 0.998$ & $95.1 / 0.997$ \\
\hline & avg. & $76.5 / 0.888$ & $71.5 / 0.839$ & $67.3 / 0.927$ & $56.7 / 0.616$ & $79.8 / 0.963$ & $94.2 / 0.989$ & $95.6 / 0.997$ \\
\hline
\end{tabular}

TABLE I: Accuracy and AUC for all the methods that have been trained only on ProGAN dataset.

\begin{tabular}{|c|c|c|c|c|c|c|c|c|}
\hline \multicolumn{2}{|c|}{ Pd@1\% / Pd@10\% } & Spec & FFD & PatchForensics & CNN-DCT & Wang2020 & no-down & proposed \\
\hline \multirow{8}{*}{$\begin{array}{l}\dot{\alpha} \\
3 \\
0 \\
3 \\
3\end{array}$} & ProGAN & $79.6 / 98.0$ & $61.3 / 94.2$ & $66.0 / 96.8$ & $1.5 / 15.0$ & $100.0 / 100.0$ & $100.0 / 100.0$ & $100.0 / 100.0$ \\
\hline & StyleGAN & $7.9 / 29.8$ & $23.8 / 56.0$ & 43.6 / 91.1 & $0.8 / 8.4$ & $49.4 / 85.0$ & $76.9 / 97.8$ & $97.3 / 100.0$ \\
\hline & StyleGAN2 & $4.1 / 17.8$ & $9.6 / 36.1$ & $26.7 / 82.3$ & $0.4 / 3.8$ & $40.6 / 82.0$ & $68.4 / 94.7$ & $77.6 / 98.5$ \\
\hline & BigGAN & $36.9 / 77.3$ & $2.2 / 15.8$ & $12.4 / 62.7$ & $1.7 / 16.6$ & $16.2 / 64.3$ & $70.5 / 97.5$ & $91.0 / 99.6$ \\
\hline & CycleGAN & $56.6 / 87.1$ & $17.3 / 50.4$ & $36.5 / 61.6$ & $1.7 / 16.7$ & $51.1 / 91.1$ & $57.6 / 90.7$ & $88.6 / 98.7$ \\
\hline & StarGAN & $0.8 / 27.2$ & $68.3 / 98.2$ & $99.8 / 100.0$ & $1.7 / 16.8$ & $65.5 / 94.6$ & $81.2 / 99.4$ & $97.9 / 100.0$ \\
\hline & RelGAN & $87.0 / 99.6$ & $43.5 / 92.7$ & $99.9 / 100.0$ & $1.7 / 16.8$ & $24.9 / 71.7$ & $52.1 / 95.7$ & $93.7 / 99.8$ \\
\hline & GauGAN & $18.9 / 61.9$ & $1.2 / 17.0$ & $1.5 / 30.9$ & $1.7 / 16.8$ & $61.3 / 93.8$ & $77.4 / 97.5$ & $89.6 / 99.5$ \\
\hline \multirow{4}{*}{ 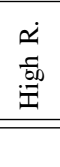 } & ProGAN & $83.2 / 88.0$ & $29.2 / 92.2$ & $100.0 / 100.0$ & $0.9 / 9.0$ & $100.0 / 100.0$ & $100.0 / 100.0$ & $100.0 / 100.0$ \\
\hline & StyleGAN & $88.5 / 96.9$ & $16.6 / 64.0$ & $51.3 / 85.3$ & $2.2 / 21.7$ & $84.5 / 98.9$ & $94.5 / 99.8$ & $99.0 / 100.0$ \\
\hline & StyleGAN2 & $98.1 / 99.8$ & $3.4 / 54.3$ & $15.9 / 69.6$ & $0.1 / 1.4$ & $50.2 / 87.6$ & $97.1 / 99.8$ & $93.2 / 99.7$ \\
\hline & avg. & $51.1 / 71.2$ & $25.1 / 61.0$ & $50.3 / 80.0$ & $1.3 / 13.0$ & $58.5 / 88.1$ & $79.6 / 97.5$ & $93.4 / 99.6$ \\
\hline
\end{tabular}

TABLE II: Pd@1\% and Pd@10\% for all the methods that have been trained only on ProGAN dataset.

augmentation and overall for our solution that achieves on the average the best performance.

\section{CONCLUSION}

We proposed a new method based on contrastive learning for GAN image detection. It generalizes well to GAN architectures never seen in training and is robust to disruptive forms of post-processing, like compression and resizing. Moreover, it represents a first step towards a universal detector. Among the many problems that remain to investigate, beyond mere performance, we want to address robustness to malicious operations, like adversarial attacks.

\section{ACKNOWLEDGEMENT}

This material is based on research sponsored by the Defense Advanced Research Projects Agency (DARPA) and the Air Force Research Laboratory (AFRL) under agreement number FA8750-20-2-1004. The views and conclusions contained herein are those of the authors and should not be interpreted as necessarily representing the official policies or endorsements, either expressed or implied, of DARPA and AFRL or the U.S. Government. In addition, this work is supported by Google and by the PREMIER project, funded by the Italian Ministry of Education, University, and Research within the PRIN 2017 program.
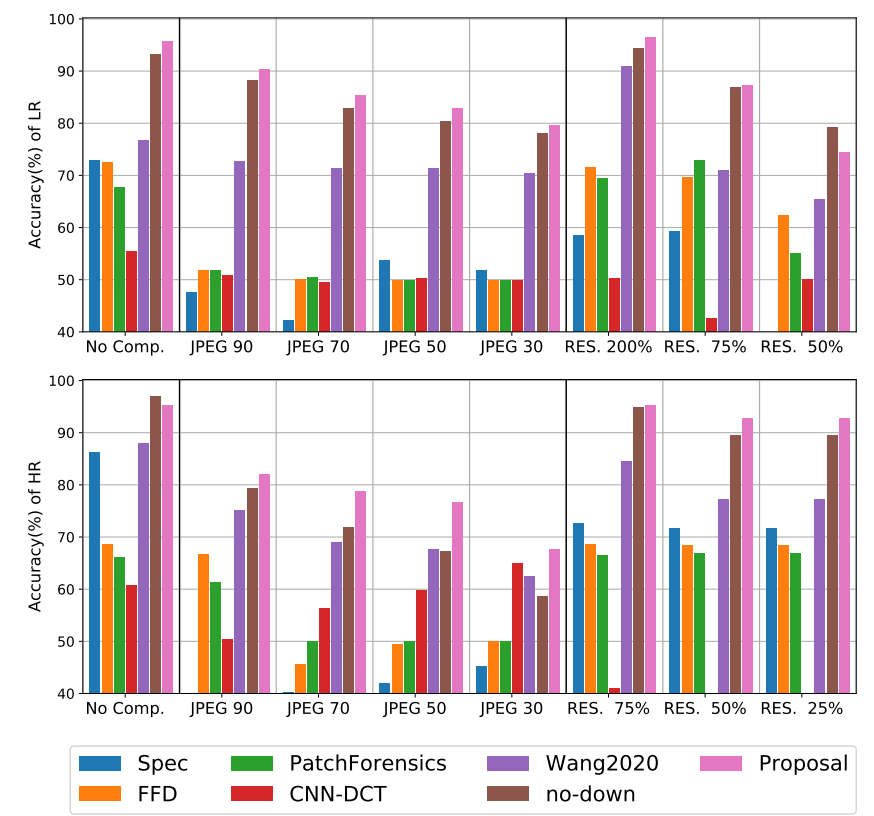

Fig. 4: Performance in terms of accuracy on low-resolution (LR) images (top) and high resolution (HR) images (bottom) by varying the JPEG compression level and rescaling the image of different factors. 


\section{REFERENCES}

[1] T. Karras, T. Aila, S. Laine, and J. Lehtinen, "Progressive Growing of GANs for Improved Quality, Stability, and Variation," in International Conference on Learning Representations (ICLR), 2018.

[2] T. Karras, S. Laine, M. Aittala, J. Hellsten, J. Lehtinen, and T. Aila, "Analyzing and improving the image quality of StyleGAN," in IEEE Conference on Computer Vision and Pattern Recognition (CVPR), 2020, pp. 8110-8119.

[3] S. Nightingale, S. Agarwal, E. Härkönen, J. Lehtinen, and H. Farid, "Synthetic faces: how perceptually convincing are they?" in Vision Sciences Society (VSS) meeting, 2021.

[4] F. Lago, C. Pasquini, R. Boehme, H. Dumont, V. Goffaux, and G. Boato, "More Real than Real: A Study on Human Visual Perception of Synthetic Faces," arXiv preprint arXiv:2106.07226v1, 2021.

[5] CNN, "A high school student created a fake 2020 candidate. Twitter verified it," Www.cnn.com/2020/02/28/tech/faketwitter-candidate-2020/ index.html

[6] N. Gleicher, "Removing Coordinated Inauthentic Behavior," https://about.fb.com/news/2020/09/removing-coordinated-inauthenticbehavior-china-philippines/ 2020.

[7] F. Marra, D. Gragnaniello, L. Verdoliva, and G. Poggi, "Do GANs leave artificial fingerprints?" in IEEE Conference on Multimedia Information Processing and Retrieval (MIPR), 2019, pp. 506-511.

[8] N. Yu, L. Davis, and M. Fritz, "Attributing Fake Images to GANs: Learning and Analyzing GAN Fingerprints," in IEEE International Conference on Computer Vision (ICCV), 2019.

[9] L. Verdoliva, "Media forensics and deepfakes: an overview," IEEE Journal of Selected Topics in Signal Processing, vol. 14, no. 5, pp. 910-932, 2020.

[10] S.-Y. Wang, O. Wang, R. Zhang, A. Owens, and A. Efros, "CNNgenerated images are surprisingly easy to spot... for now," in IEEE Conference on Computer Vision and Pattern Recognition (CVPR), 2020

[11] H. Dang, F. Liu, J. Stehouwer, X. Liu, and A. K. Jain, "On the detection of digital face manipulation," in IEEE Conference on Computer Vision and Pattern Recognition (CVPR), June 2020.

[12] L. Chai, D. Bau, S.-N. Lim, and P. Isola, "What makes fake images detectable? Understanding properties that generalize," in European Conference on Computer Vision (ECCV), 2020, pp. 103-120.

[13] X. Zhang, S. Karaman, and S.-F. Chang, "Detecting and Simulating Artifacts in GAN Fake Images," in IEEE Workshop on Information Forensics and Security (WIFS), 2019, pp. 1-6.

[14] J. Frank, T. Eisenhofer, L. Schönherr, A. Fischer, D. Kolossa, and T. Holz, "Leveraging frequency analysis for deep fake image recognition," in International Conference on Machine Learning (ICML), 2020 pp. 3247-3258.

[15] D. Cozzolino, J. Thies, A. Rössler, C. Riess, M. Nießner, and L. Verdoliva, "ForensicTransfer: Weakly-supervised domain adaptation for forgery detection," arXiv preprint arXiv:1812.02510, 2018.

[16] F. Marra, C. Saltori, G. Boato, and L. Verdoliva, "Incremental learning for the detection and classification of GAN-generated images," in IEEE International Workshop on Information Forensics and Security (WIFS), 2019, pp. 1-6.

[17] X. Xuan, B. Peng, W. Wang, and J. Dong, "On the generalization of GAN image forensics," in Chinese Conference on Biometric Recognition, 2019, pp. 134-141.

[18] D. Gragnaniello, D. Cozzolino, F. Marra, G. Poggi, and L. Verdoliva, "Are GAN generated images easy to detect? A critical analysis of the state-of-the-art," in IEEE International Conference on Multimedia and Expo (ICME), 2021, pp. 1-6.

[19] Y. Yousfi, J. Butora, E. Khvedchenya, and J. Fridrich, "ImageNet Pretrained CNNs for JPEG Steganalysis," in IEEE Workshop on Information Forensics and Security (WIFS), 2020.

[20] F. Marra, D. Gragnaniello, L. Verdoliva, and G. Poggi, "A full-image full-resolution end-to-end-trainable $\mathrm{CNN}$ framework for image forgery detection," IEEE Access, vol. 8, 2020.

[21] T. Chen, S. Kornblith, M. Norouzi, and G. Hinton, "A simple framework for contrastive learning of visual representations," in International Conference on Machine Learning, vol. 119, 2020, pp. 1597-1607.

[22] P. Khosla, P. Teterwak, C. Wang, A. Sarna, Y. Tian, P. Isola A. Maschinot, C. Liu, and D. Krishnan, "Supervised contrastive learning," in Advances in Neural Information Processing Systems (NIPS), vol. 33,2020 , pp. 18661-18673.
[23] D. P. Kingma and J. Ba, "Adam: A method for stochastic optimization," in International Conference on Learning Representations (ICLR), 2015.

[24] T. Karras, S. Laine, and T. Aila, "A style-based generator architecture for generative adversarial networks," in IEEE Conference on Computer Vision and Pattern Recognition (CVPR), 2019, pp. 4396-4405.

[25] A. Brock, J. Donahue, and K. Simonyan, "Large Scale GAN Training for High Fidelity Natural Image Synthesis," in International Conference on Learning Representations (ICLR), 2018.

[26] J.-Y. Zhu, T. Park, P. Isola, and A. Efros, "Unpaired image-to-image translation using cycle-consistent adversarial networks," in International Conference on Computer Vision (ICCV), 2017.

[27] Y. Choi, M. Choi, M. Kim, J.-W. Ha, S. Kim, and J. Choo, "StarGAN: Unified generative adversarial networks for multi-domain image-toimage translation," in IEEE Conference on Computer Vision and Pattern Recognition (CVPR), 2018, pp. 8789-8797.

[28] P.-W. Wu, Y.-J. Lin, H. C. C, E. Chang, and S.-W. Liao, "RelGAN: Multi-domain image-to-image translation via relative attributes," in International Conference on Computer Vision (ICCV), 2019.

[29] T. Park, M.-Y. Liu, and T.-C. W. J.-Y. Zhu, "Semantic image synthesis with spatially-adaptive normalization," in IEEE Conference on Computer Vision and Pattern Recognition (CVPR), 2019, pp. 2337-2346.

[30] T.-Y. Lin, M. Maire, S. Belongie, L. Bourdev, R. Girshick, J. Hays, P. Perona, D. Ramanan, C. L. Zitnick, and P. Dollar, "Microsoft COCO: Common objects in context," in European Conference on Computer Vision (ECCV), 2014.

[31] D.-T. Dang-Nguyen, C. Pasquini, V. Conotter, and G. Boato, "RAISE: A raw images dataset for digital image forensics," in ACM Multimedia Systems Conference, 2015, pp. 219-224. 